\title{
An Examination of FORMOSAT-3/COSMIC Ionospheric Electron Density Profile: Data Quality Criteria and Comparisons with the IRI Model
}

\author{
Kuo-Feng Yang ${ }^{1}$, Yen-Hsyang $\mathrm{Chu}^{1,{ }^{*}}$, Ching-Lun $\mathrm{Su}^{1}$, Hsiao-Tsun $\mathrm{Ko}^{1}$, and Chien-Ya Wang ${ }^{2}$ \\ ${ }^{1}$ Institute of Space Science, National Central University, Chung-Li, Taiwan, ROC \\ ${ }^{2}$ Department of Physics, Chinese Culture University, Taipei, Taiwan, ROC
}

Received 30 April 2007, accepted 5 October 2007

\begin{abstract}
In this article, we analyze the properties of ionospheric electron density profiling retrieved from FORMOSAT-3/COSMIC radio occultation measurements. Two parameters, namely, the gradient and fluctuation of the topside electron density profile, serve as indicators to quantitatively describe the data quality of the retrieved electron density profile. On the basis of 8 month data (June 2006 - January 2007), we find that on average 93\% of the electron density profiles have upper electron density gradients and electron density fluctuations smaller than $-0.02 \# / \mathrm{m}^{3} / \mathrm{m}$ and 0.2 , respectively, which can be treated as good data for further analysis. The same results are also achieved for the peak height of the electron density. After removing the questionable data, we compare the general behaviors of the electron density between FORMOSAT- 3 and the IRI model. It is found that the global distributions of the peak height and the peak electron density for the FORMOSAT-3/COSMIC data are generally consistent with those for the IRI model. However, a significant difference between their scale heights of the topside electron density profiles is found. It suggests that the shape of the topside electron density profile in the IRI model should be revised accordingly such that it more closely resembles the real situation.
\end{abstract}

Key words: IRI model, Peak height, Peak electron density

Citation: Yang, K. F., Y. H. Chu, C. L. Su, H. T. Ko, and C. Y. Wang, 2009: An examination of FORMOSAT-3/COSMIC ionospheric electron density profile: Data quality criteria and comparisons with the IRI model. Terr. Atmos. Ocean. Sci., 20, 193-206, doi: 10.3319/TAO.2007.10.05.01(F3C)

\section{INTRODUCTION}

Radio occultation technique is an old, but very sophisticated, method for the retrieval terrestrial atmosphere parameters (Fjeldbo et al. 1971). The core of this technique is (under a number of assumptions) to transform the bending angle of the radio ray path to the atmospheric refractive index, which is transmitted from a very stable source situated on one side of the Earth and received by a receiver located on the opposite side of the Earth (Rocken et al. 1997; Hajj et al. 2000). Once the atmospheric refractive index is retrieved, the lower atmospheric temperature, humidity and ionospheric electron density at the tangent point of the ray path piercing through the atmosphere can be estimated in accordance with the relation between the refractive index $n$ and the parameters given below:

\footnotetext{
* Corresponding author

E-mail:yhchu@jupiter.ss.ncu.edu.tw
}

$\mathrm{n}=1+\left(\frac{77.6 \mathrm{P}}{\mathrm{T}}+\frac{373000 \mathrm{e}}{\mathrm{T}^{2}}-\frac{5.6 \mathrm{e}}{\mathrm{T}}\right) \times 10^{-6}-\frac{40.3 \mathrm{n}_{\mathrm{e}}}{\mathrm{f}^{2}}$

where $\mathrm{P}$ is pressure (hpa), T is temperature (k), e is water vapor pressure (hpa), $\mathrm{f}$ is radio wave frequency $(\mathrm{Hz})$, and $\mathrm{n}_{\mathrm{e}}$ is electron density $\left(\# / \mathrm{m}^{3}\right)$. In the ionosphere (higher than an altitude of about $100 \mathrm{~km}$ ), the contribution of T, P and e to the atmospheric refractive index is negligible compared to the electron density contribution. As a result, $\mathrm{n}_{\mathrm{e}}$ can be directly estimated from $\mathrm{n}$ for given $\mathrm{f}$. Except for the atmospheric refractive index, under the straight line assumption of the radio ray path, the height variation of the ionospheric electron density can also be retrieved from calibrated total electron content (TEC) in accordance with the Abel transformation, which can be estimated from the phase path difference between L1 (= $1.57542 \mathrm{GHz})$ and L2 (= $1.22760 \mathrm{GHz})$ frequencies of GPS signals (Schriner et al. 1999). 
A constellation of 6 small satellites, called FORMOSAT-3/COSMIC, was successfully launched on 15 April 2006. Three payloads, namely, 4 sets of GPS signal receivers, a Tri-band (150, 400, and $1067 \mathrm{MHz})$ beacon transmitter system, and the tiny ionospheric photometer at $135.6 \mathrm{~nm}$, are on board each of the COSMIC small satellites for weather/climate, ionosphere and geodesy researches (Rocken et al. 2000). The ionospheric electron densities employed in this study are measured by GPS receivers with radio occultation technique, which can obtain nearly 2500 profiles of lower atmospheric temperature, humidity, and ionospheric electron density in 24 hours. The advantages of the FORMOSAT-3/COSMIC data are high resolution (about $1 \mathrm{~km}$ ) in vertical and quasi-uniform distribution with resolution about $250-300 \mathrm{~km}$ in horizontal around the globe. Establishment of the appropriate data quality control algorithm for the selection of good quality data from the raw dataset is absolutely necessary for further applications of FORMOSAT-3/COSMIC data.

One of the most critical assumptions made in the retrieval of the radio-occultated atmospheric parameters based on the Abel transformation is the spherical symmetry of the atmospheric refractive index (Hajj et al. 2000; Kursinski et al. 2000). This assumption implies that no horizontal gradient of the refractive index exists along the spherical shell. In addition, geometric optics assumption is also a crucial condition for GPS radio occultation inversion (Kursinski et al. 2000). With this assumption, the GPS radio wave propagation can be modeled by a ray path and the scattering effect of the refractive index irregularities on the GPS signal can be ignored (Born and Wolf 1980). Consequently, because of no irregular electron density distributions in the GPS ray path, it is expected that the occultation-retrieved electron density profile will be a smooth curve without random fluctuations superimposed on the curve. However, if there are electron density irregularities existing in the ray path, the scintillation and multiple paths effects will disturb the received GPS signals. As a result, the retrieved electron density profile will be highly fluctuating due to irregular variation in the bending angle of the ray path. In this article, the global behavior of the random fluctuation of the electron density profile will be studied. We will show that the global distribution of the electron density fluctuation is highly seasonal and latitude dependent.

The accuracy and precision of the radio-occultated electron density has been validated by using measurements made by ground-based ionosondes and incoherent scatter radars (ISR) (Hajj and Romans 1998; Rius et al. 1998; Hajj et al. 2000; Tsai et al. 2001; Jakowski et al. 2002; Lei et al. 2007). A long-term comparison between GPS/MET and ionosonde peak electron densities made by Hajj et al. (2000) indicates that, under solar minimum condition, the fractional difference in the peak hourly electron density between GPS/ MET and ionosonde is about $20-40 \%$, which is defined as the ratio of the difference between GPS/MET and ionosonde hourly peak electron densities to the corresponding hourly average of the mean electron density of the GPS/MET. Hocke and Igarashi (2002a) compared peak electron densities of the sporadic E (Es) layer at noon retrieved by GPS/MET satellite with those measured by ionosondes distributed in the Eastern Asia Section and found that they are in general agreement with each other. Stolle et al. (2004) compared the high-latitude electron density profiles between CHAMP and EISCAT measurements and found that the occultation technique apparently tends to overestimate the electron density when the background electron densities are low. Except for the data measured by the ionosonde and ISR, the radio-occultated electron densities were also compared with the model predictions. Hocke and Igarashi (2002b) compared meridional electron density cross-sections observed by GPS/MET with those predicted by IRI2001 and found that they gave a satisfactory agreement. However, significant departures are detected for the nighttime topside ionosphere at low latitudes and for the southern polar winter ionosphere. They also observed that the GPS/ MET data show lower electron densities in the upper F region in equatorial and low latitude regions than IRI-2001 predictions, and the peak height of the polar F-layer observed by GPS/MET is generally lower by about $50-100 \mathrm{~km}$ than that predicted by the IRI model. In order to obtain correct electron density profile, Lei et al. (2007) used scale height at $\mathrm{F}$ peak height and correlation coefficient between fitted and observed profiles to serve as the rejection criteria to screen out the COSMIC electron density data such that the COSMIC measurements with good quality are employed to compare with ground-based observations, IRI and TIEGCM model predictions.

It is noteworthy that most of the validations of the radio-occultated ionospheric electron densities focus on the values around peak and bottom side of the electron density profile. To the best of authors' knowledge, very limited studies on the property of the electron density profile above the peak have been reported. For example, Stankov and Jakowski (2006) analyzed the scale height of topside electron density profiles retrieved by CHAMP satellite, which characterizes the plasma temperature and the diffusion behavior of the topside electron density. They found that the scale height generally increases poleward, particularly during equinox and summer, and there is a tendency for the summer daytime scale heights in mid-latitude region to be higher than those in winter. In this research, the general behavior of the scale height of the electron density above the peak height retrieved by the COSMIC satellites will be investigated. We find that there are significant differences in the general behaviors, such as diurnal and seasonal variations and latitudinal and longitudinal distributions, of the scale heights between FORMOSAT-3/COSMIC ionospheric measurements and International Reference Ionosphere (IRI) model prediction. 
The organization of this article is as follows: In section 2 , the thresholds for the data quality control of the COSMIC-measured electron density profiles are set up to screen out the bad and/or questionable data. In section 3, we compare the general behaviors of the COSMIC-deduced electron density profiles with those computed by the IRI mode. We especially focus on the comparisons of the behaviors of peak electron densities and peak height in the F2-region, and the scale height of the topside electron density profiles between COSMIC ionospheric measurements and IRI model results. Finally, a discussion is given and conclusions drawn in section 4.

\section{THRESHOLDS FOR DATA QUALITY CONTROL}

Many factors may influence the accuracy and precision of the electron density retrieved from the occultation inversion, including random fluctuations and steep gradients of the plasma density existing in the GPS ray path, system bias, noises generated by the hardware and retrieval algorithm, inaccurate position of the satellites, and so on, leading to erroneous estimation of the bending angle of the GPS ray. As a result, retrieved electron density will be fluctuated and deviated from the true one, causing the retrieval results to be questionable. Figure 1 demonstrates examples of normal (left panel) and questionable (right panel) COSMIC electron density profiles, in which large and irregular spikes and a salient data gap are shown in the questionable profiles. Obviously, the questionable profiles should be removed before further analysis and application.

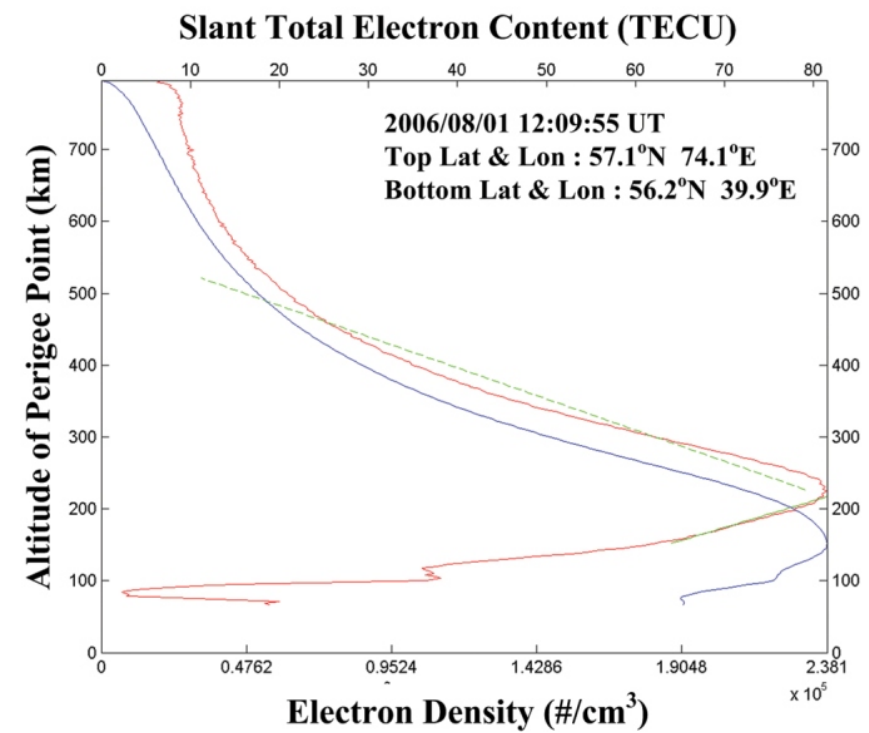

\subsection{Mean Deviation of Electron Density Fluctuation}

It is noteworthy that large fluctuation in the height variation of the electron density may be a physically meaningful phenomenon and should not be treated as meaningless noise or erroneous data. The use of two or more COSMIC satellites that are close enough in the orbits with very small spatial separation to simultaneously receive the GPS signal transmitted from the same GPS satellite provides us an opportunity to examine whether the fluctuations in the electron density profile are meaningful or not. Figure $2 \mathrm{com}$ pares the electron density (red curve) and calibrated TEC (blue curve) profiles measured by satellite number 3 (right panel) and 4 (left panel), respectively, which received the GPS signal transmitted from GPS satellite number 11 at almost the same time (only a 14-second difference in the two). In addition, the differences in the longitudes and latitudes between the tangent points of the GPS rays for FM3 and 4 are very small, less than $0.043^{\circ}$ and $0.273^{\circ}$, respectively. As shown, in spite of exceedingly irregular fluctuations of the height variations of the electron densities, we find perfectly one-to-one correspondence of the peaks of the random fluctuations in these two electron density profiles. Furthermore, the magnitudes of the peaks are also comparable to each other. Because of remarked similarity between these two profiles, we consider that the fluctuations in the height variations of the electron densities are very likely to be physically real and result from electron density irregularities occurrring in the GPS ray path.

In order to quantitatively assess the effect of the iono-

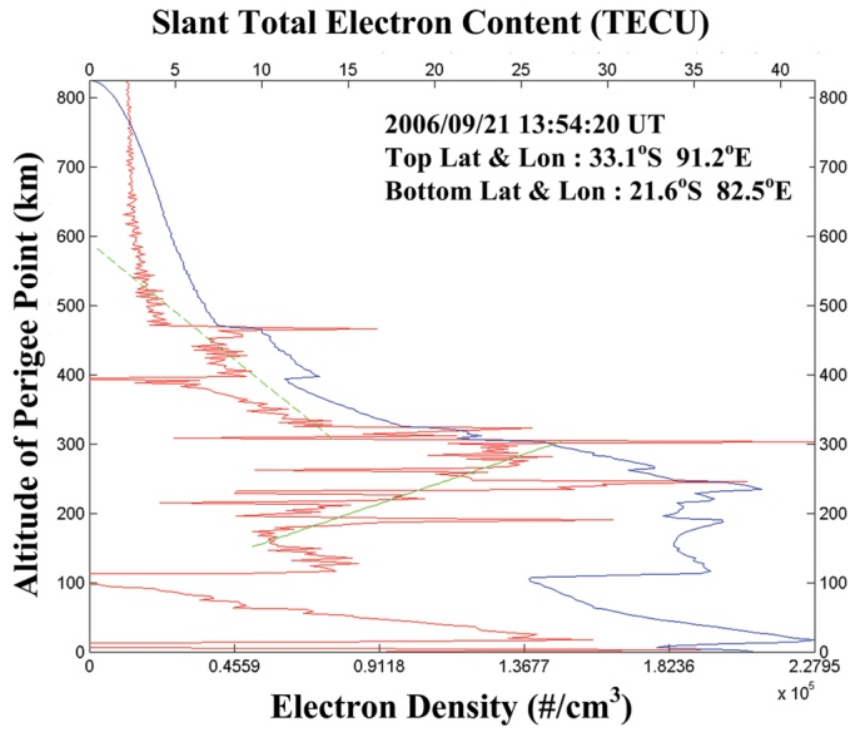

Fig. 1. Two examples of questionable electron density profiles, in which large and irregular spikes (right panel) and a salient data gap (left panel) are shown in the respective profiles. The red and blue curves are, respectively, the electron density profile and calibrated total electron content along the GPS ray path. The green lines are the slopes of the electron density profiles for the height ranges above and below the peak height. The COSMIC satellite that obtained the electron density and TEC profiles is FORMOSAT-3 number 5. 
FM 4

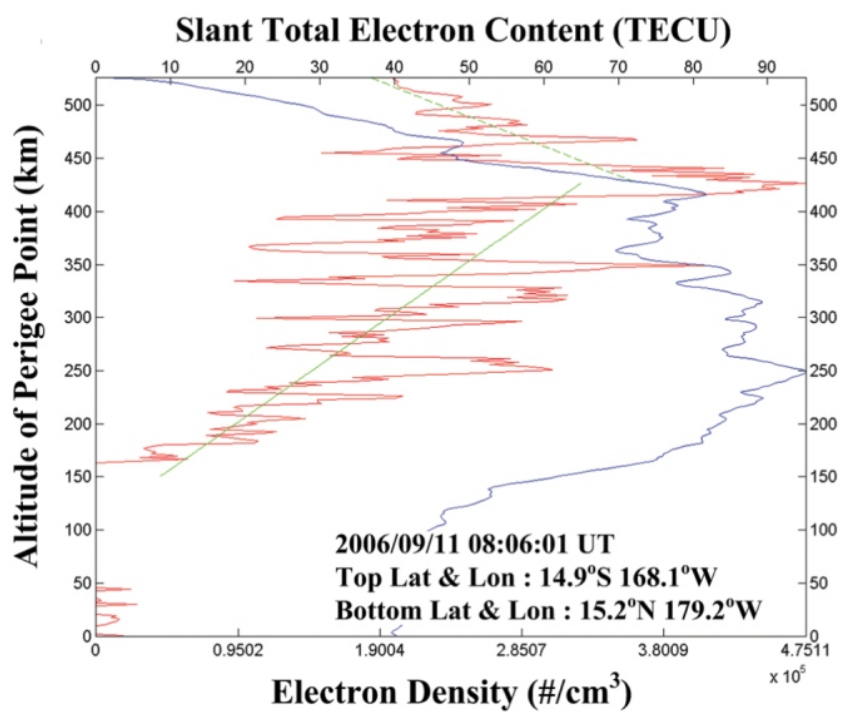

FM 3

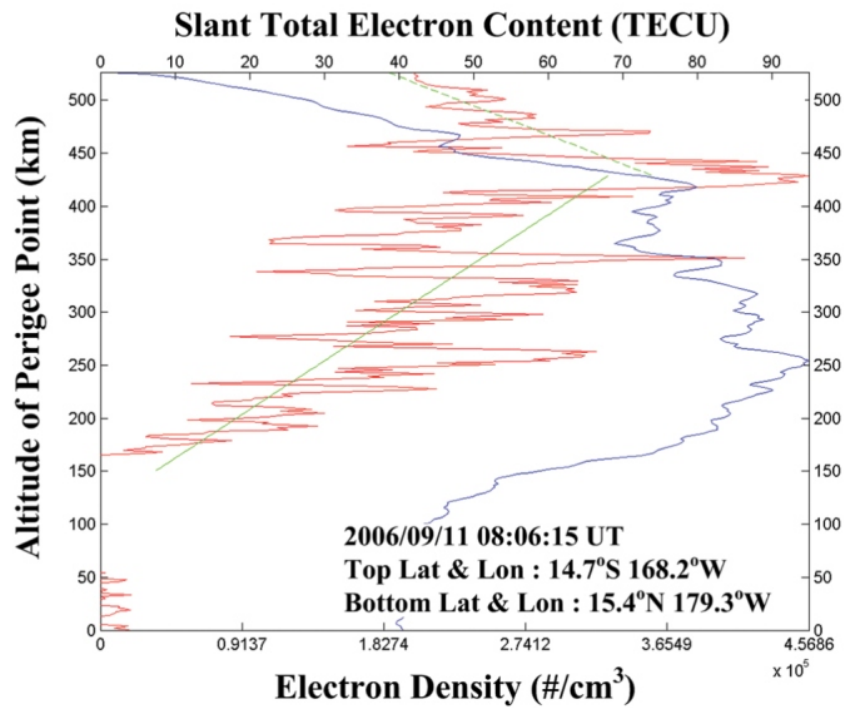

Fig. 2. A comparison of the electron density (red curve) and calibrated TEC (blue curve) profiles measured by satellite number 3 (right panel) and 4 (left panel), respectively, which received the GPS signal transmitted from GPS satellite number 11 at almost the same time (only a 14-second difference in the two). The green lines are the same as Fig. 1.

spheric plasma irregularities on the height variation of the electron density, we define mean deviation (MD) of the electron density profile as follows:

$\mathrm{MD}=\sum_{\mathrm{i}} \frac{\left|\mathrm{n}_{\mathrm{ei}}-\overline{\mathrm{n}_{\mathrm{ei}}}\right|}{\mathrm{N}_{\mathrm{ei}}}$

where $\mathrm{N}$ is the total data points in a profile, $\mathrm{n}_{\mathrm{ei}}$ and $\overline{\mathrm{n}_{\mathrm{ei}}}$ are, respectively, the measured and background electron densities at the $\mathrm{i}$-th height. Note that $\overline{\mathrm{n}_{\mathrm{ei}}}$ is obtained by taking 9-point running average of the measured electron density profile. Therefore, the farther the measured electron density deviates from the background value, the larger the magnitude of MD will be. Except for the assessment of the plasma irregularity effect, MD can also be employed for data quality control. For questionable data shown in Fig. 1, extraordinarily large spikes appearing in the electron density profile will make the magnitude of MD enormously large. Therefore, it is indispensable to set up a threshold for MD to screen out such questionable data (i.e., extraordinarily large spikes). Statistics show that almost $99 \%$ of the MDs appear in the range $0-1.5$ and the MDs are approximately uniformly distributed in the accumulative distribution as their values are grater than 1.5 , as shown in the bottom panels of Fig. 3. Therefore, the threshold for the value of MD that we set up in this research for screening out the questionable data is 1.5 .

Figure 4 depicts the global distributions of magnitudes of the MD in different seasons, in which geomagnetic equator (purple curve) and geographic equator (light green line) are both shown. As indicated, in general, the fluctuations of the electron density profiles in the winter hemisphere (i.e., January for northern hemisphere and July for southern hemisphere) are much more salient than those in the summer hemisphere (i.e., July for northern hemisphere and January for southern hemisphere). The higher the latitude is, the larger the fluctuation in the electron density profile will be. Especially notice that enormously large disturbances of the electron density profiles occur over the winter polar region where the latitude is greater than about $70^{\circ}$, and the profiles are very quiet in the summer polar region. This feature strongly suggests that the ionospheric electron densities in the winter polar region are much more irregular than those in the summer polar region. Note that the large MD in winter polar region may also be the result of relatively smaller electron density compared to the summer polar region. In addition, there is a tendency for the electron density profiles with salient fluctuations to occur in the geomagnetic equator region in the equinox season, especially in the Southern American sector where the geomagnetic equator is bended toward the south first and then the north. This region is called Southern American Anomaly (SAA) in the geomagnetic field. Obviously, it is conceivable that the irregular fluctuation of the electron density profile is associated with the ionospheric plasma irregularities that make the spherical symmetry invalid in the retrieval of the ionospheric refractive index from the bending angle of the GPS ray in accordance with Abel transformation. As a result, the stronger the electron density irregularity is, the larger the fluctuation in the electron density profile will be. Therefore, the mean fluctuation of the height variation of the electron density can be considered to be an indicator of the intensity of the electron 

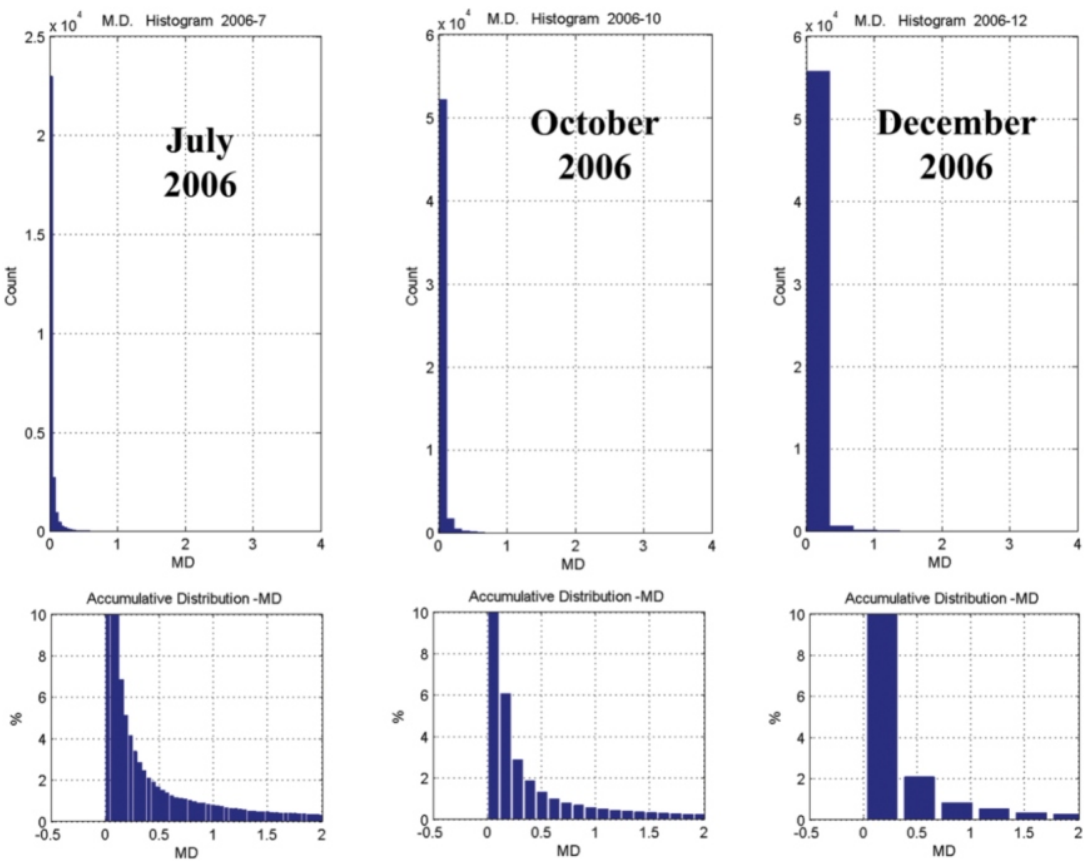

Fig. 3. Statistics of the mean deviation (MD) for different months, in which the upper panels are the histograms and the lower panels are the cumulative probability of the MD.
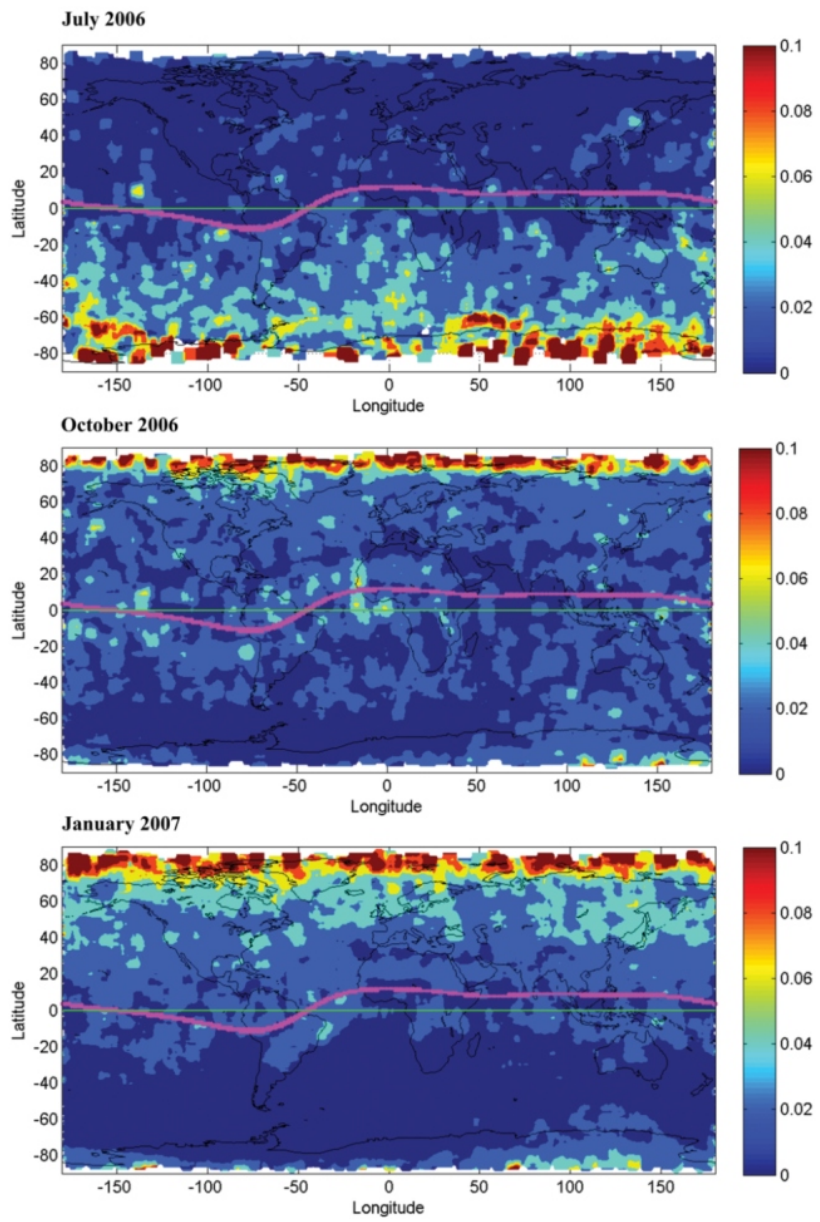

Fig. 4. Global distributions of the mean deviations of the electron density profiles in different seasons, in which geomagnetic equator (purple curve) and geographic equator (light green line) are both shown. density irregularities occurring in the GPS ray path.

\subsection{Slope of Topside $(420$ - $490 \mathrm{~km})$ Electron Density Profile}

Aside from the enormously large spikes, we note that a number of electron density profiles show positive gradient (i.e., electron density increases with increasing height) or nearly uniform distribution of the electron density in the topside portion (above the peak electron density) of the profile. Figure 5 demonstrates two examples of the COSMIC electron density profiles with positive or zero gradients. Statistics show that this type of the profile constitutes roughly $4 \%$ of all the profiles, which may appear at every location around the world. Figure 6 compares the statistics of the slope of the electron density profile in the height range 420 - $490 \mathrm{~km}$ between COSMIC measurement and IRI model for different months. As shown, except for the slopes greater than $-0.2 \# / \mathrm{m}^{3} / \mathrm{m}$, the ranges of the COSMIC-measured slopes are in general in agreement with those of the IRI model slope spanning from -0.2 to $-7 \# / \mathrm{m}^{3} / \mathrm{m}$. Therefore, aside from the threshold of MD, we select this slope range as the other threshold for the COSMIC data quality control.

The distributions of the slope are presented in Fig. 6. It is clearly shown that means and the variances between the COSMIC and IRI slope distributions are different. In addition, we note that the secondary peaks not only appear in the COSMIC slope distributions, but also occur in the IRI model slope distributions. In order to explore the cause of this secondary peak, we plot the global distribution of the slope 

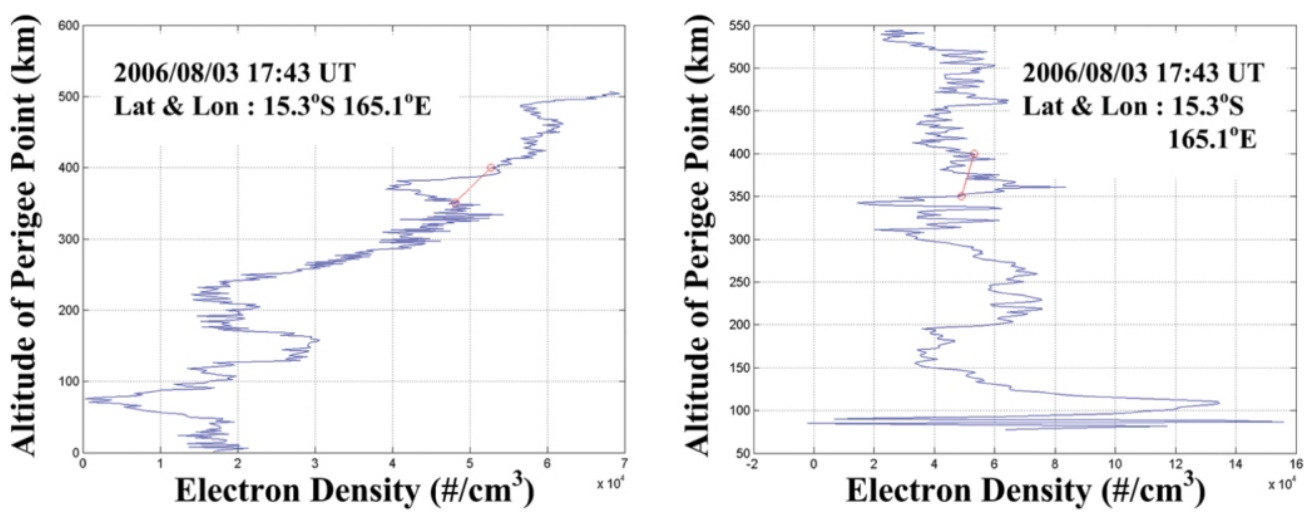

Fig. 5. Two examples of the COSMIC electron density profiles with positive (left panel) or near zero (right panel) gradients of the height variations of the electron density.
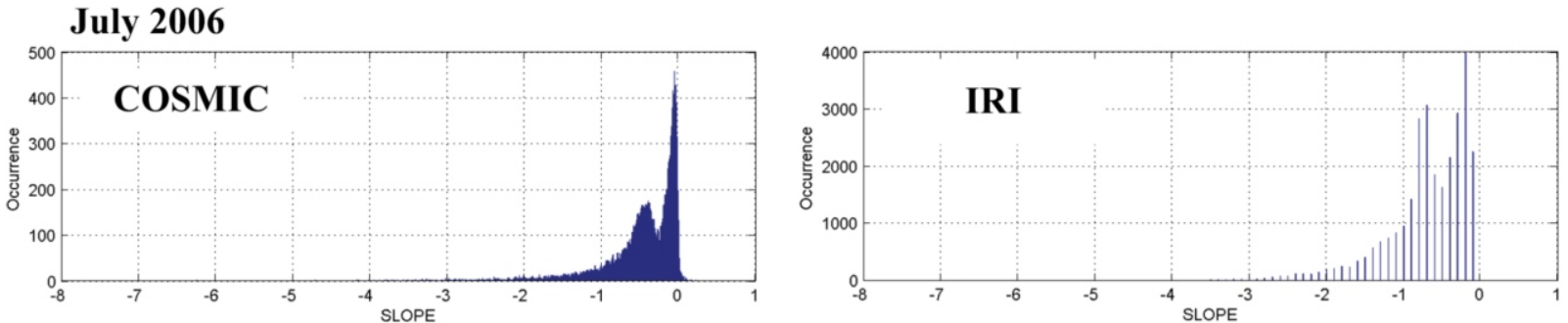

\section{October 2006}
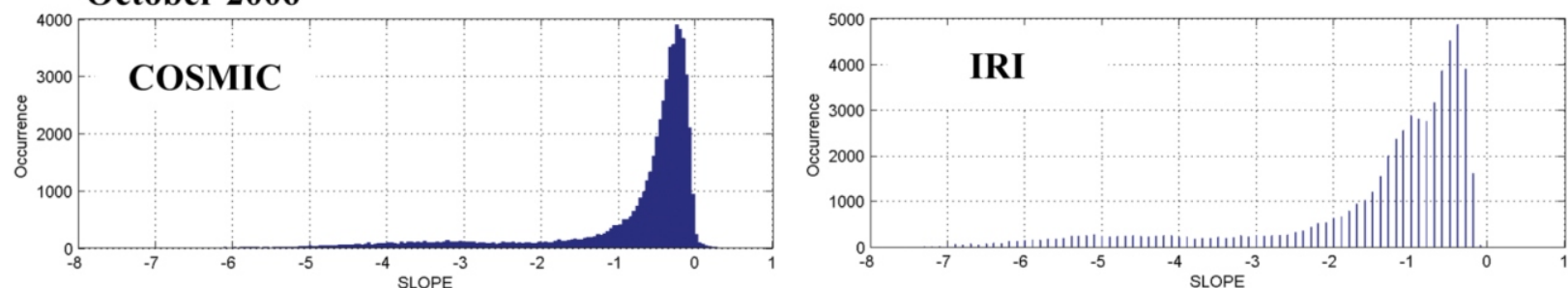

December 2006
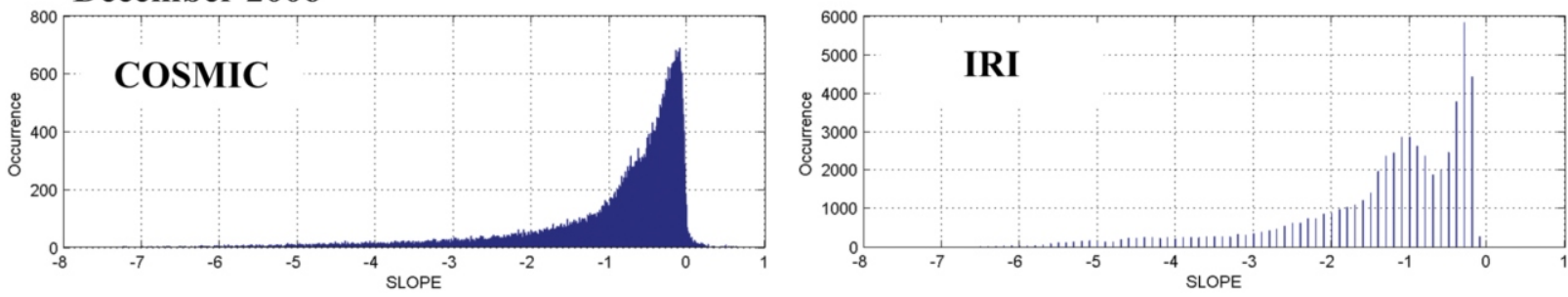

Fig. 6. A comparison of the statistics of the slope of the electron density profile in the height range 420 - $490 \mathrm{~km}$ between COSMIC measurement and the IRI model for different months.

and the results are shown in Fig. 7. It is obvious that there is a pronounced diurnal variation of the slope in the latitudinal region within $40^{\circ}$, and the diurnal variation is not obvious above $40^{\circ}$. The absolute values of the slope are larger in daytime time than those in nighttime, with peaks occurring during 1300 - 1500 LT. Comparing the histogram shown in Fig. 6 with the global distributions of the slope presented in Fig. 7 reveals that the secondary peak in the slope histogram is the result of the daytime bulges of the slope, which is more pronounced in winter than that in summer. In addition to the diurnal variation, there is a remarked seasonal variation in the slope of the upper electron density profile. The absolute values of the slopes in mid- and high latitudes in wintertime are smaller than those in summer time. Moreover, the peak location of the slope varies with the season; it locates in northern (southern) hemisphere in summer (winter) season. 

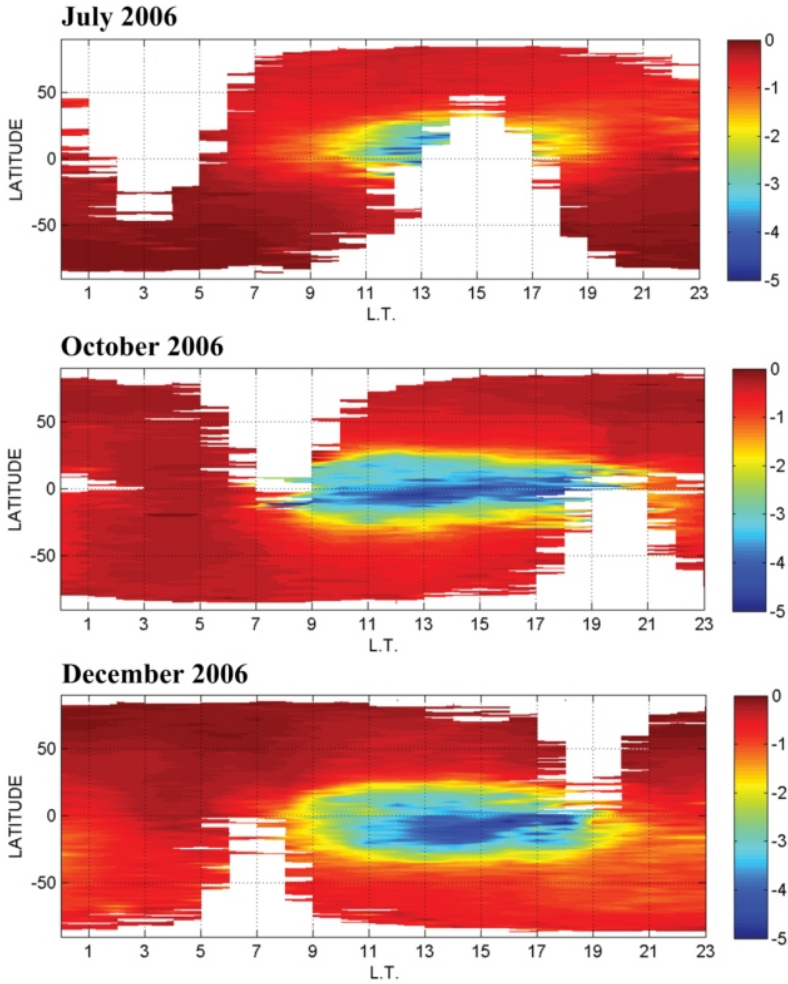

Fig. 7. Global distributions of the COSMIC-measured slope for different seasons.

\section{COMPARISON BETWEEN COSMIC MEASUREMENT AND THE IRI MODEL}

In this section, we compare the general behaviors, including global distribution, seasonal and diurnal variations, of the electron density profiles retrieved by the COSMIC measurements and predicted by the IRI model. Figure 8 compares the global distributions of the monthly averaged peak electron densities between COSMIC data (left panels) and IRI model predictions (right panels), in which the peak electron density is the daily mean value. As shown, the global behaviors of the peak electron densities predicted by the IRI model are generally consistent with those observed by FORMOSAT-3/COSMIC satellites, including seasonal variations, equatorial anomaly, and the synoptic-scale pattern of the peak electron density distributed along the geomagnetic equator. Except for the globally spatial distributions, the diurnal variations of the peak electron densities observed by the COSMIC satellites and predicted by the IRI model are also compared and the results are presented in Fig. 9. It is obvious that, regardless of the slight difference between their values, the general behaviors of the diurnal variations of the peak electron densities in different seasons obtained by the COSMIC satellites bear a strong resemblance to those given by the IRI model. The latitudinal ex-

\section{COSMIC Peak Electron Density}

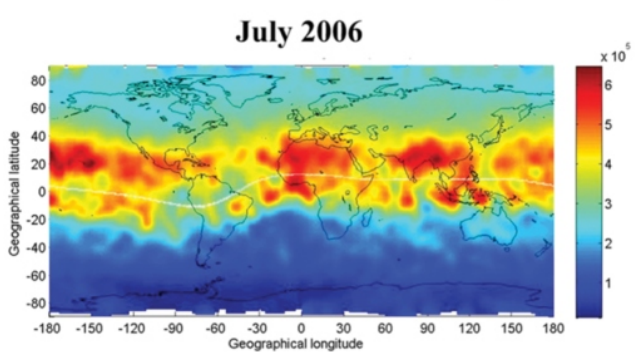

October 2006

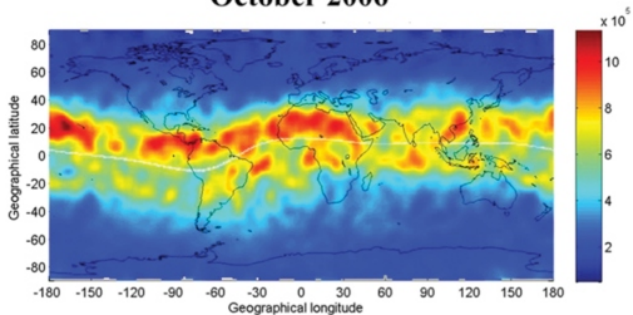

January 2007

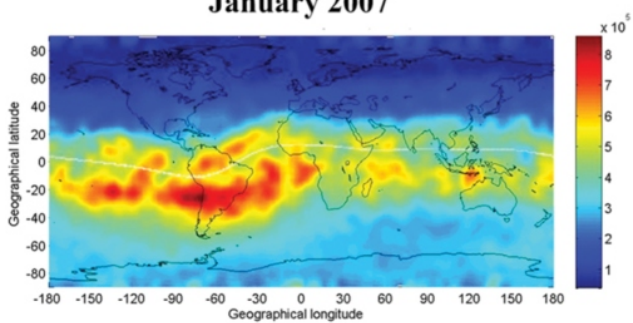

\section{IRI Peak Electron Density}

July 2006

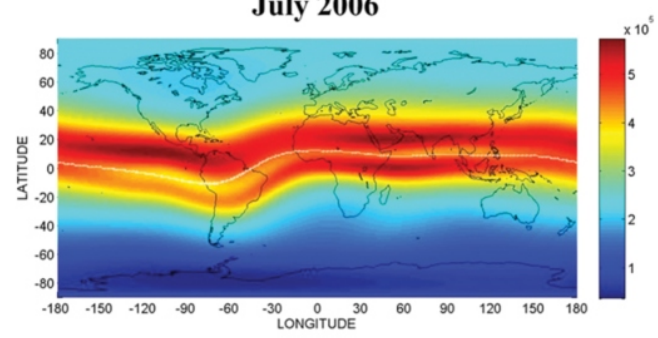

October 2006

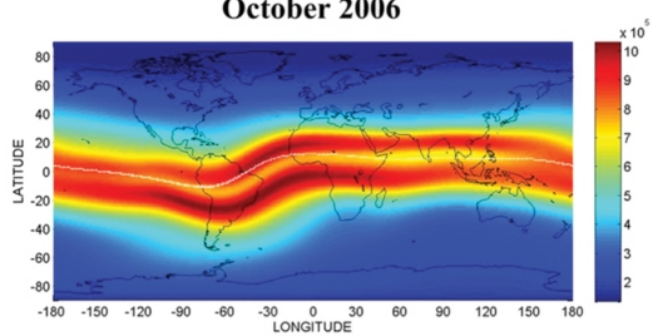

January 2007

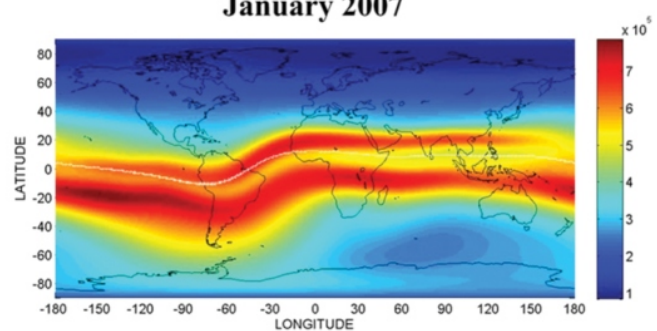

Fig. 8. A comparison of the global distributions of the monthly averaged peak electron densities between COSMIC data (left panels) and IRI model predictions (right panels). 
COSMIC Peak Electron Density

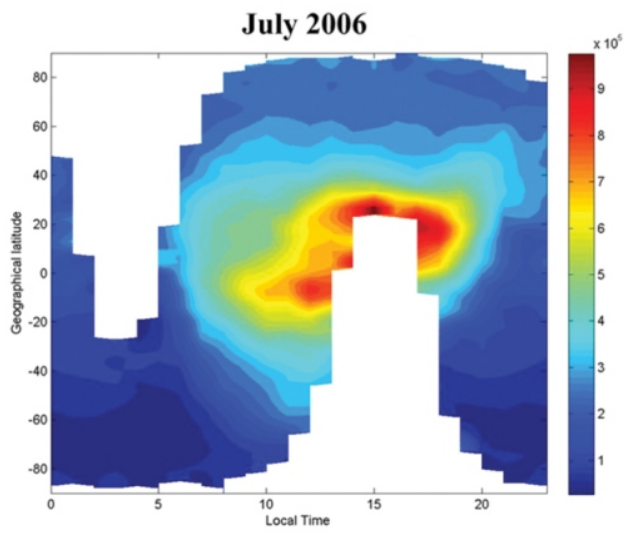

October 2006

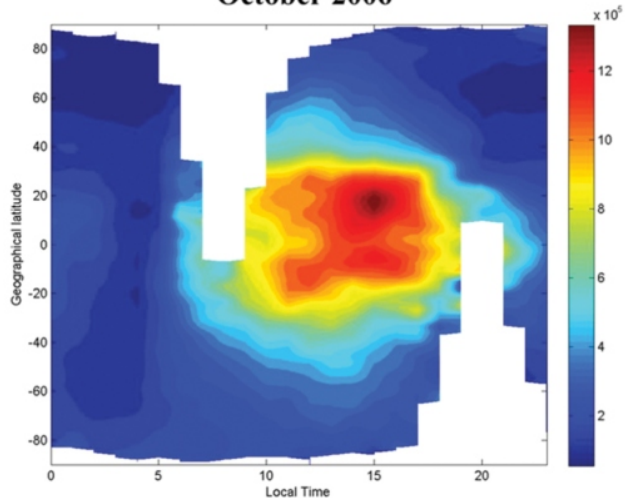

January 2007

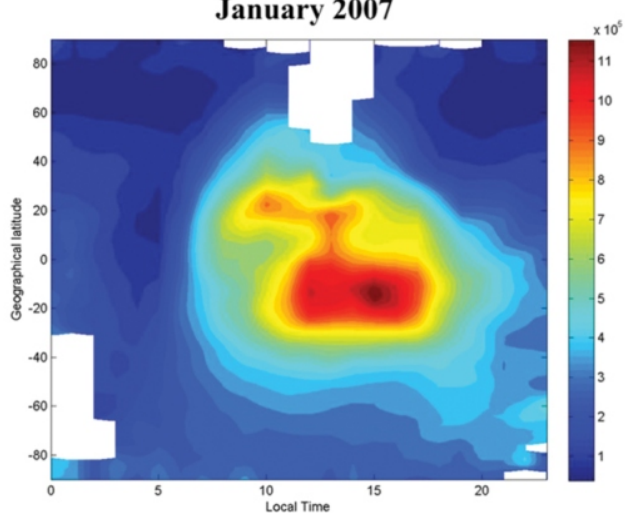

IRI Peak Electron Density
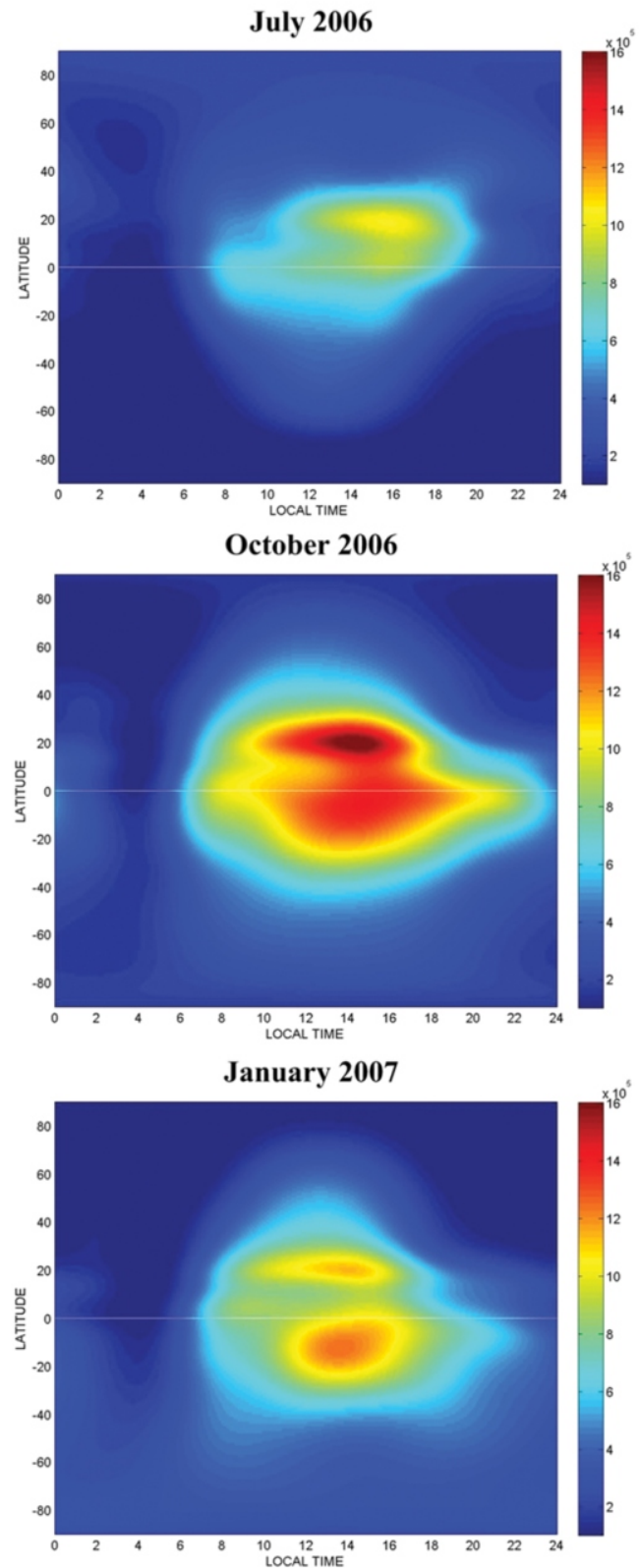

Fig. 9. Latitude-local time variation of the monthly mean peak electron densities for different seasons obtained by FORMOSAT-3/COSMIC measurements (left panels) and IRI model predictions (right panels).

tents and the seasonal variations of the equatorial anomaly seen in the COSMIC measurements are in agreement with those from the IRI model.

Figure 10 compares the global distributions of the monthly averaged peak heights of the electron density profiles between COSMIC data (left panels) and IRI model predictions (right panels), in which the data are also the daily average. As shown, the COSMIC and IRI peak heights are both maximum over the geomagnetic equator region and relatively lower in the mid- and high latitude areas. Although the tendencies for the global distributions of the COSMIC and IRI peak heights are generally similar, large discrepancies are found in the intensity and width of the equatorial ridge. Moreover, as indicated in Fig. 10, it is evident both from IRI and COSMIC data that the peak height seems to have a longitudinal variation along the geomagnetic equator. Namely, the maximum values tend to occur in the regions of west of India, west of South Africa, east-south Asia and mid-pacific Ocean, respectively. This longitudinal wavenumber-4 structure has been observed from the spatial distributions of total 
COSMIC Peak Height

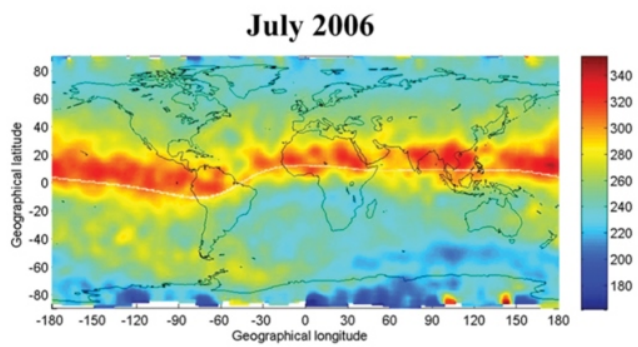

October 2006

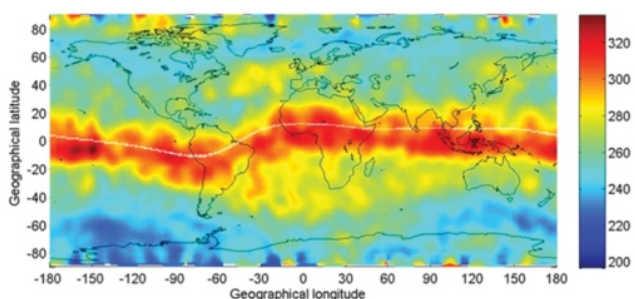

January 2007

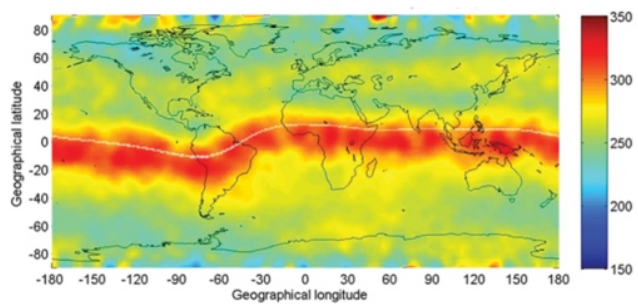

IRI Peak Height

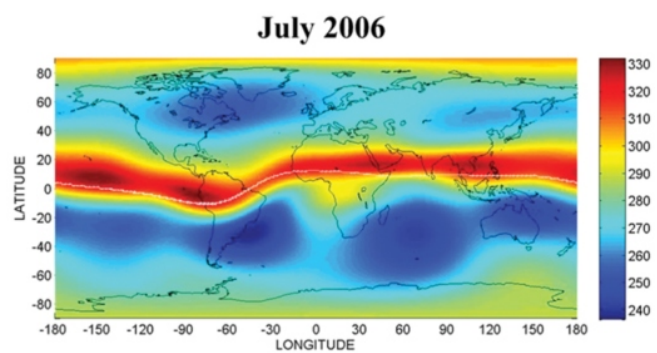

October 2006

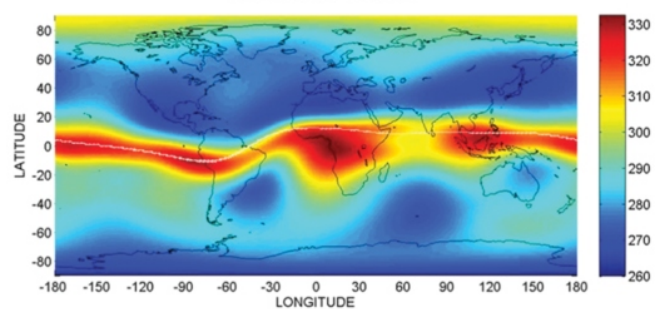

January 2007

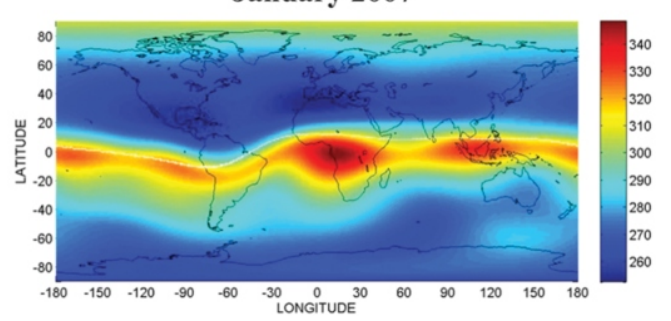

Fig. 10. A comparison of the global distributions of the monthly averaged peak heights between COSMIC data (left panels) and IRI model predictions (right panels).

electron content (TEC) and far-ultraviolet (FUV) 135.6-nm emission from the $5 \mathrm{~S}-3 \mathrm{P}$ transition of $\mathrm{O}^{+}$ion, which was made by the IMAGE satellite (Vladimer et al. 1999; Immel et al. 2006). This feature is very likely associated with the non-migrating diurnal atmospheric tides that are driven mainly by weather in the lower troposphere in the tropics (Immel et al. 2006).

Figure 11 shows the latitude-local time variation of the monthly mean peak heights for different seasons obtained by FORMOSAT-3/COSMIC measurements (left panels) and IRI model predictions (right panels). As indicated, during the daytime the peak heights are maximum over the equatorial region and minimum in the mid-latitude area. However, during the nighttime the situations are opposite, minimum over the equator and maximum in the mid-latitude regions. In addition, from Fig. 11 irrespective of the seasonal variation in the locations of the equatorial maxima of the peak heights not being obvious, the peak height itself shows a strongly seasonal dependence. Namely, the COSMIC measurements and the IRI model results both show that the peak heights are larger in summer than those in winter. Moreover, equatorial anomaly is absent in the latitudinal variation of the peak height. Therefore, from the results shown in Figs. 8 - 11 , it summarizes that the general behaviors of the radio- occultated electron density profiles observed by COSMIC satellites are in good agreement with those predicted by IRI model. The similar results are also achieved by comparing the ionopsheric data retrieved by other radio occultation experiments, such as Ionopsheric Occultation Experiment (IOX), with the ionopsheric climatology data from the RIGB model (Straus 2007).

As mentioned above, although the IRI model can basically describe the general behaviors of the COSMIC-measured peak electron density and the peak heights, we will show evidence later that it is not the case for the scale height of the topside electron density profile. The scale height $\mathrm{H}$ that we estimate from the electron density profile is obtained by best fitting a simple exponential function to the observed data in the height range $420-490 \mathrm{~km}$, which is in the following form:

$\mathrm{n}_{\mathrm{e}}(\mathrm{z})=\mathrm{n}_{\mathrm{e} 0} \mathrm{e}^{-(\mathrm{z}-420) / \mathrm{H}}$

where $\mathrm{n}_{\mathrm{e} 0}$ is the electron density at height $420 \mathrm{~km}$. Note that the scale height defined in this article is different from that defined by Stankov and Jakowski (2006). We will discuss the difference in the two in section 4. 


\section{COSMIC Peak Height}

July 2006

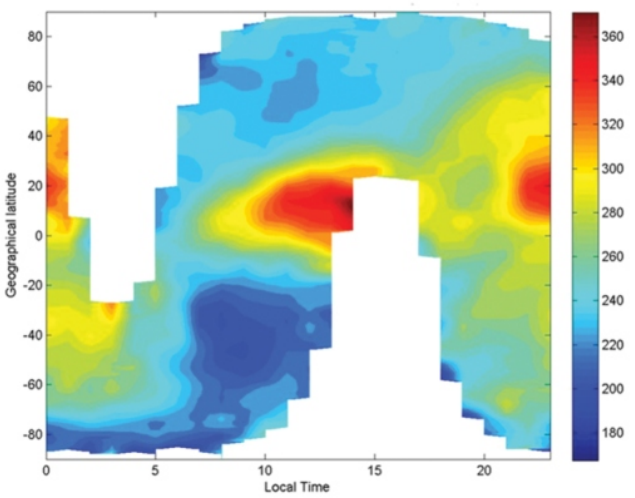

October 2006

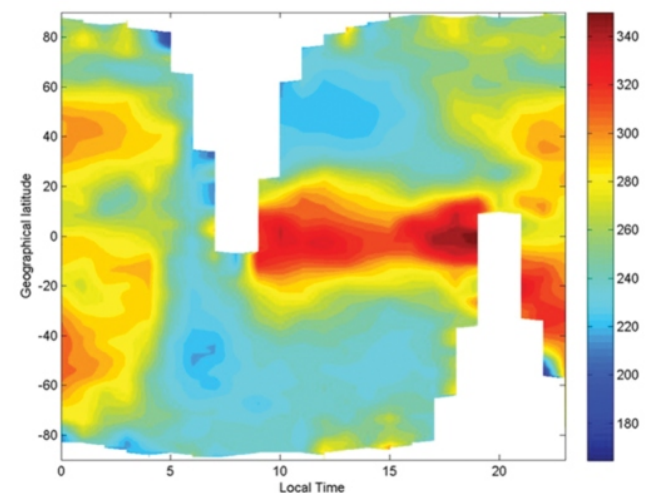

January 2007

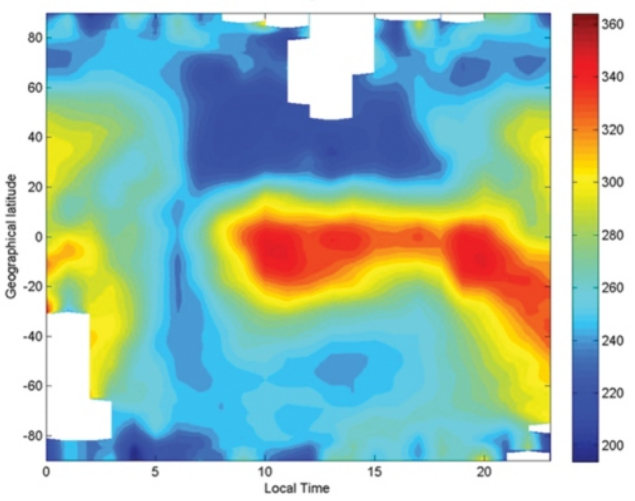

IRI Peak Height

July 2006

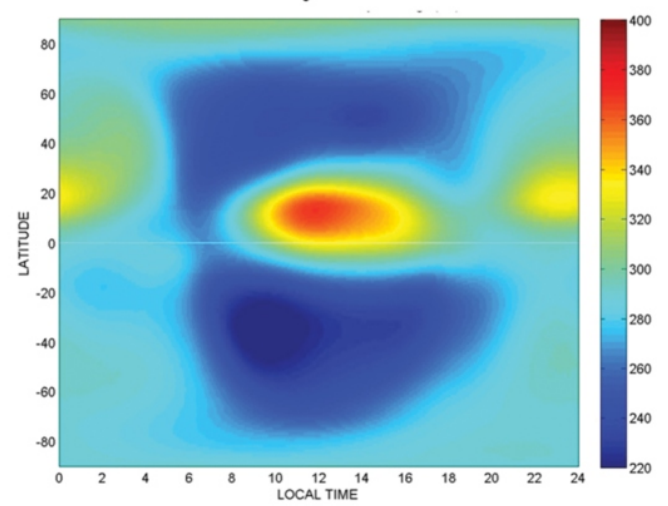

October 2006

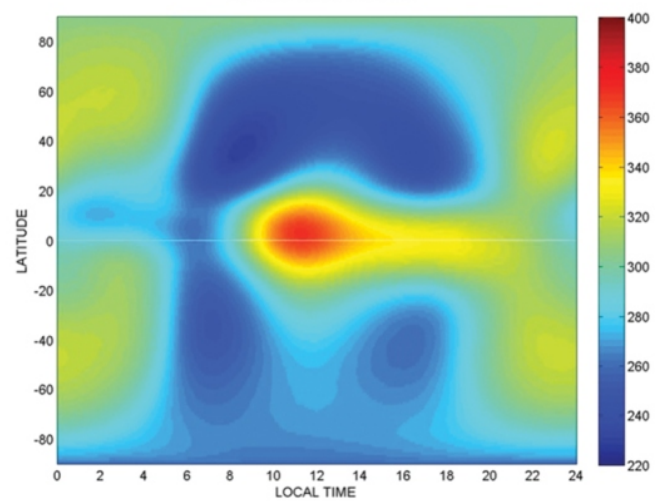

January 2007

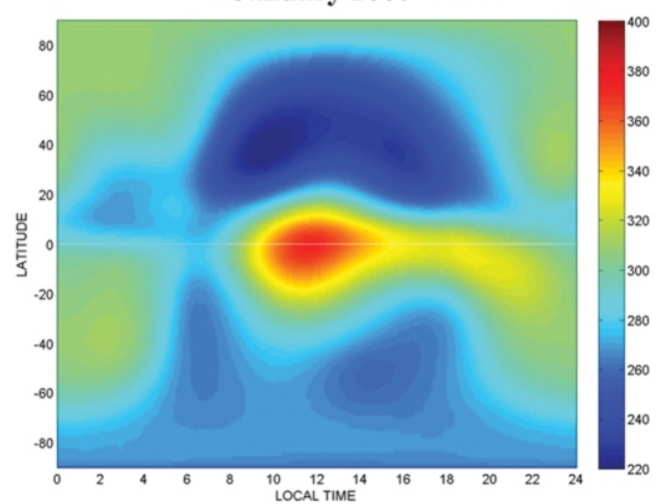

Fig. 11. Latitude-local time variation of the monthly mean peak heights for different seasons obtained by FORMOSAT-3/COSMIC measurements (left panels) and IRI model predictions (right panels).

Figure 12 compares the global distributions of the monthly averaged scale heights estimated from topside (height range $420-490 \mathrm{~km}$ ) electron density profiles between COSMIC data and the IRI model, in which the scale heights for different seasons (i.e., summer, equinox, and winter) are shown. It is obvious from Fig. 10 that the COSMIC results are very different from the IRI model results. The IRI model predicts that, irrespective of the seasons, the peaks of the monthly mean scale heights are distributed along the geomagnetic equator with meridional width of approximately $\pm 20^{\circ}$. In mid- and high latitude regions, the IRI-predicted scale heights are much smaller than those in the equatorial region. However, the global distribution of the COSMIC-deduced scale height is totally different from that predicted by the IRI model. As shown in the left panels of Fig. 12, the global behavior of the scale height of the topside electron density profile is strongly season-dependent. The scale heights in high latitude regions are much greater than those in midlatitude and equator regions, although there is a tendency for weak peaks of the scale heights to appear over 


\section{Scale Height for COSMIC Data}

July 2006

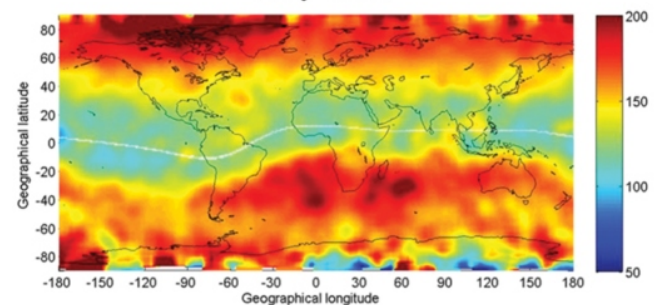

October 2006

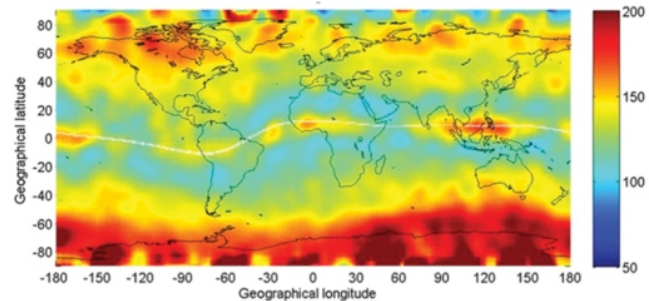

January 2007

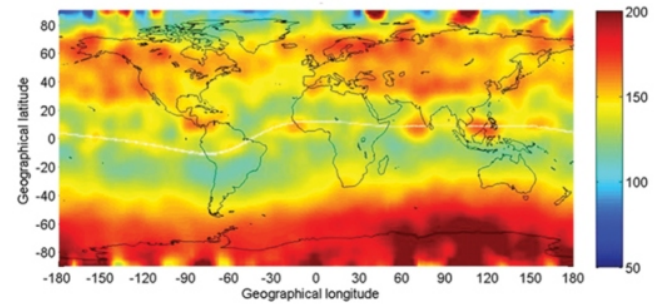

Scale Height for IRI Model

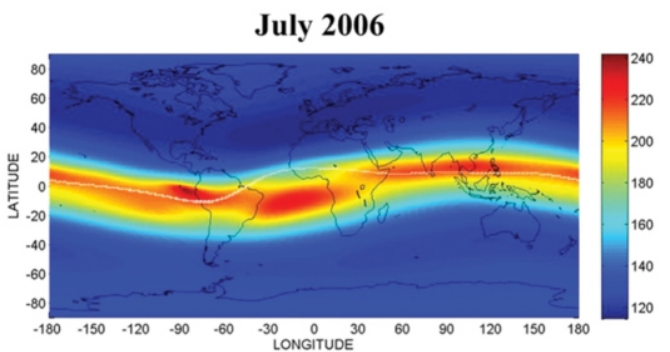

October 2006

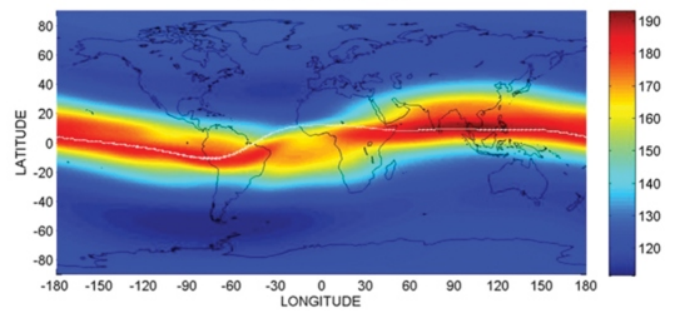

January 2007

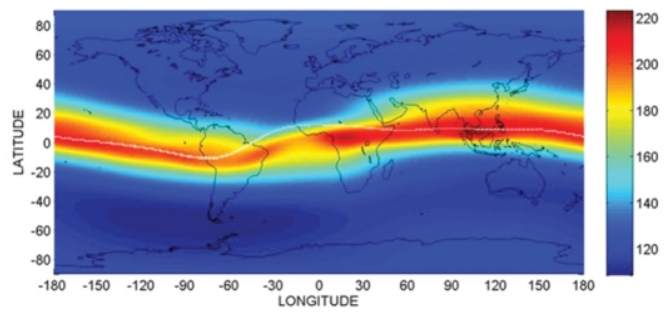

Fig. 12. Global distributions of the monthly averaged scale heights estimated from topside (height range 420 - $490 \mathrm{~km}$ ) electron density profiles between COSMIC data and the IRI model, in which the scale heights for different seasons (i.e., summer, equinox and winter) are shown.

the geomagnetic equator in equinox and winter. We also note that in wintertime the observed scale heights in the high latitude region in the southern hemisphere are much larger than those in the northern hemisphere, and vice versa for those in summertime.

Except for the monthly averaged data, the diurnal variations of the scale height for COSMIC and IRI model results are also very different. As indicated in the right panels of Fig. 13, the IRI model predicts that a remarkable peak of the scale height occurs at the time around sunrise over the equator region. In addition, IRI model also predicts that during the daytime the scale height will be greater in equator region than those in mid- and high latitude regions. However, these features cannot be seen in the COSMIC-measured data. Therefore, a comparison of the scale heights between COSMIC and IRI model results as presented in Fig. 13 which suggest that the properties of the topside electron density profile of the IRI model do not meet the observational results and should be significantly revised for better performance in ionospheric predictions.

\section{DISCUSSION AND CONCLUSION}

As shown in Fig. 7, the magnitude of the electron den- sity fluctuation in the polar region depicts a remarked seasonal variation, which is much more intense during winter (December for northern hemisphere) than summer (July for northern hemisphere). This feature is very consistent with the observations of the UHF scintillation activity reported by Aarons et al. (1981). They found that the maximum occurrence of the scintillation in the high latitude region, which is associated with the ionospheric plasma irregularities generated either by the charged particle precipitation or by plasma instability (Aarons 1982), appears in months of little or no sunlight (namely, winter season) at $\mathrm{F}$ region heights, and much lower scintillation occurrence appears in sunlight months (namely, summer season). In addition, Aarons et al. (1981) also showed that the diurnal variation of the scintillation is weak in the high latitude region during the winter season. Therefore, it suggests that MD of the electron density profile seems to be able to serve as an indicator of the degree of electron density fluctuations associated with plasma irregularities.

A comparison of peak values and peak heights of the electron density profiles between COSMIC-measured and IRI-predicted data shows that the behaviors of the global distributions and diurnal and seasonal variations of the COSMIC 
Scale Height for COSMIC Data

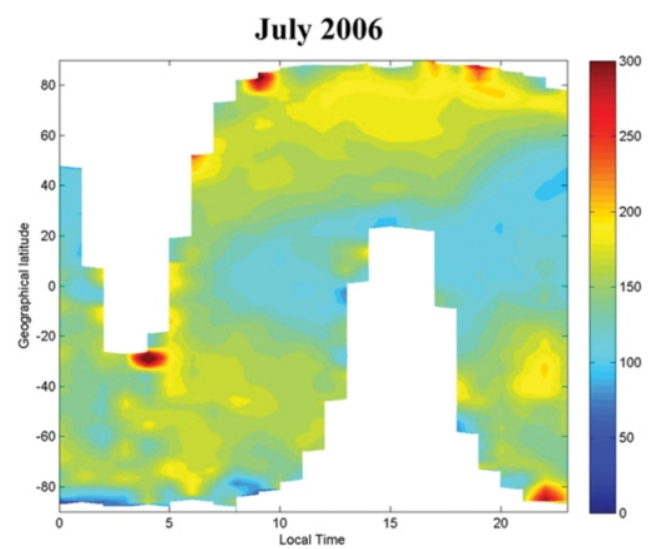

October 2006

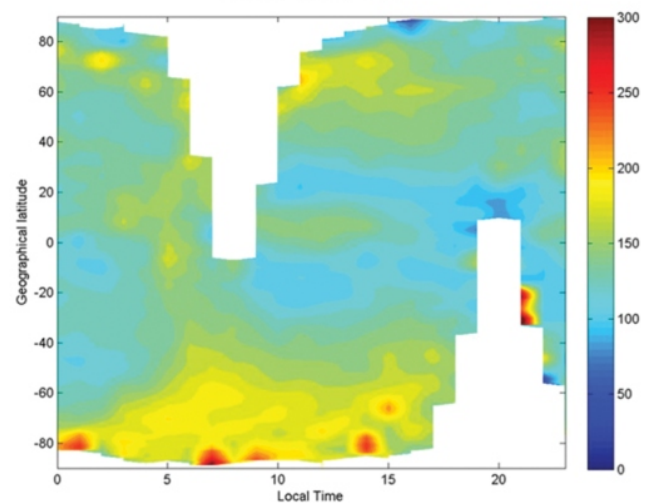

January 2007

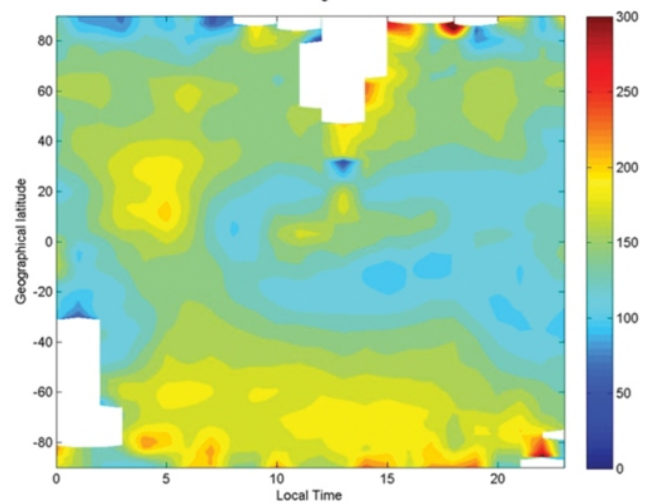

Scale Height for IRI Model

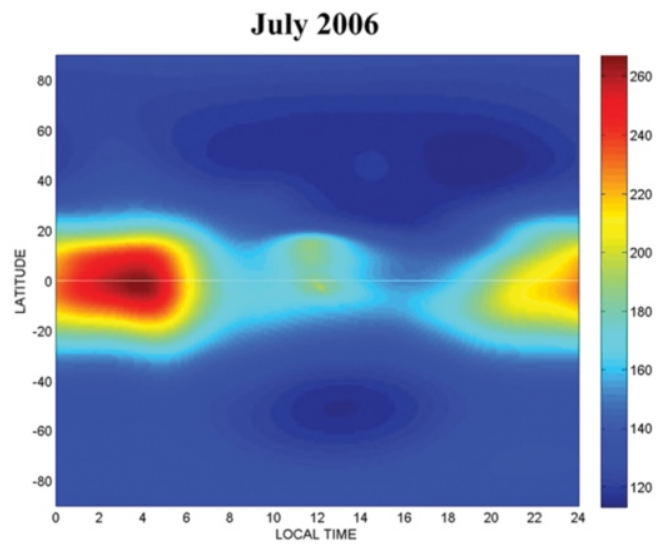

October 2006
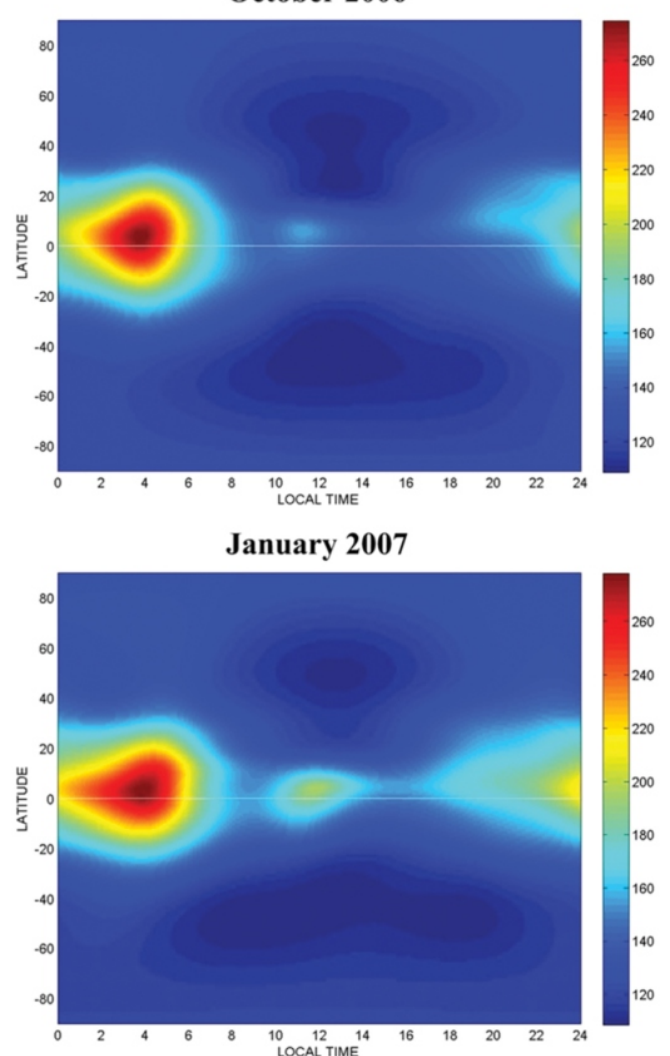

Fig. 13. Latitude-local time variation of the monthly mean scale heights for different seasons obtained by FORMOSAT-3/COSMIC measurements (left panels) and IRI model predictions (right panels).

and IRI model data are both in good agreement with each other. However, for the scale height deduced from the topside electron density profile in a height range of $420-490 \mathrm{~km}$, the difference between COSMIC and IRI model data is remarkably large. The scale heights at mid- and high latitudes from COSMIC data strongly depend on the season at regions, while their seasonal variation predicted by the IRI model is much smaller. In addition, large discrepancies in the diurnal variations and global distributions of the scale heights in the equatorial region between COSMIC observations and IRI model predictions are also seen. Therefore, great caution is advised when the IRI model is intended to be used to describe the behavior of the upper part of the electron density profile.

On the basis of the definition of the scale height, mathematically the scale height can be re-written as:

$H=-\left(\frac{1}{n_{e}} \frac{\mathrm{dn}_{e}}{d z}\right)^{-1}$

Assume that the ionosphere is in an equilibrium state, 
that is, the accelerations of ion and electron are both equal to zero. In this condition, the pressure gradient force, gravity force, collision between charged (ion and electron) and neutral particles, electric force and Lorentz force acting on the charged particles are balancing each other. If we consider only diffusion along the geomagnetic line that is assumed to be vertical, we have (Rishbeth and Garriott 1969):

$\frac{1}{\mathrm{H}}=\frac{1}{\mathrm{H}_{\mathrm{p}}}+\frac{\mathrm{W}_{\mathrm{D}}}{\mathrm{D}}+\frac{\mathrm{d} \ln \mathrm{T}_{\mathrm{p}}}{\mathrm{dz}}$

where $\mathrm{W}_{\mathrm{D}}$ is the vertical diffusion velocity of charged particle; $\mathrm{D}$ is the diffusion coefficient and is given by $\mathrm{D}=$ $2 \mathrm{kTp} / \mathrm{m}_{\mathrm{i}} v_{\mathrm{in}}$; Tp is the plasma temperature defined as $\mathrm{Tp}=$ $(\mathrm{Te}+\mathrm{Ti}) / 2$; $\mathrm{Te}$ and $\mathrm{Ti}$ are, respectively, electron and ion temperatures; $v_{\text {in }}$ is the collision frequency between ion and neutral particles; and Hp is the plasma scale height defined as $\mathrm{Hp}=\mathrm{kTp} / \mathrm{m}_{\mathrm{i}} \mathrm{g}$; $\mathrm{k}$ is the Boltzman constant; and $\mathrm{m}_{\mathrm{i}}$ is the ion mass. From Eq. (5), although a number of physical parameters determine the magnitude of $\mathrm{H}$, the long-term statistics of the measurements made by Arecibo incoherent scatter radar show that in general approximately $90 \%$ of the magnitude of $\mathrm{H}$ or more is contributed by $\mathrm{Hp}$, and other parameters play a very minor roles in governing $\mathrm{H}$ (Liu et al. 2007).

Except for the scale height defined by Eq. (4), there are other ways to define the scale height of the topside electron density profile. For example, Stankov and Jakowski (2006) used an electron density model to describe the topside electron density profile, which is given by (Rishbeth and Garriott 1969):

$\mathrm{n}_{\mathrm{e}}(z)=\mathrm{n}_{\mathrm{m}} \exp \left\{\frac{1}{2}[1-\mathrm{Z}-\exp (-\mathrm{Z})]\right\}+\mathrm{n}_{\mathrm{p}} \exp \left(-\frac{\mathrm{z}}{\mathrm{H}_{\mathrm{P}}}\right)(6)$

where $\mathrm{Z}$ is the reduced height defined as $\mathrm{Z}=\left(\mathrm{z}-\mathrm{h}_{\mathrm{m}} \mathrm{F}_{2}\right) / \mathrm{Hc}$; $n_{m}$ is the peak electron density; $h_{m} F_{2}$ is the peak height; $H_{P}$ is the plasma scale height in the plasmasphere; and $n_{P}$ is the electron density at the plasmasphere basis defined as the $\mathrm{H}^{+}-\mathrm{O}^{+}$transition height situated at height between $800-$ $1400 \mathrm{~km}$ (Stankov et al. 2003). According to the definition as shown in Eq. (6), Stankov and Jakowski (2006) found that there is no common pattern for the diurnal variations: sometimes daytime values are higher, sometimes the night-time values dominate; large differences are detected from season to season and from latitude to latitude. Generally, the scale height increases at higher latitudes, although a few exceptions do exist. Obviously, their results are very different from the results obtained in this study, as shown in Figs. 10 and 11. It is noteworthy from Eq. (6) that the scale height defined by Stankov and Jakowski (2006) is based on $\alpha$-Chapman layer model, which bears a strong relation to the neutral temperature and density in the topside ionosphere (Rishbeth and Garriott 1969). Therefore, the scale height defined in this research $\mathrm{H}$ is much more representative of the plasma behaviors of the topside electron density than that defined by $\alpha$-Chapman model Hc proposed by Stankov and Jakowski (2006). In fact, on the basis of electron density profiles measured by Arecibo ISR, Liu et al. (2007) found that the magnitude of $\mathrm{H}$ is considerably greater than Hc by a factor of around 3. Therefore, the use of Hc will under-estimate the true scale height of the topside electron density profile.

Notice that the plasma scale height in the topside ionosphere - from the peak height (i.e., $\mathrm{h}_{\mathrm{m}} \mathrm{F}_{2}$ ) of electron density profile in $\mathrm{F}$ region up to the $\mathrm{O}^{+}-\mathrm{H}^{+}$ion transition level, where the plasmasphere starts, is very difficult to obtain (Stankov and Jakowski 2006). This is because conventionally the observed data of the topside electron density profile made by ISRs and satellites are very rare. However, the advent of the GPS radio occultation inversion technique can provide a large amount of the topside electron density data and the characteristics of the topside electron density profile will be eventually revealed.

In summary, we set up in this article the thresholds for the quality control of radio-occultated electron density profiles to screen out questionable data, in which the mean deviation and the topside slope of the electron density profile are introduced. With these data quality control thresholds, we analyze the global distributions and seasonal and diurnal variations of the peak height and maximum electron density of the COSMIC-measured profiles. After comparison with IRI model predictions, we find that they are generally consistent with each other. However, large differences in topside scale heights between COSMIC and IRI electron density profiles exist, including global distribution and seasonal and diurnal variations. This suggests that the IRI model should be updated to better characterize the general behavior of the topside electron density profile.

Acknowledgements This work was partially supported by National Space Organization (NSPO) of the Republic of China in Taiwan under grant NSC 95-2111-M-008-008.

\section{REFERENCES}

Aarons, J., 1982: Global morphology of ionospheric scintillation. Proc. IEEE, 70, 360-378, doi: 10.1109/PROC.1982. 12314. [Link]

Aarons, J., J. P. Mullen, H. E. Whiteny, A. L. Johnson, and E. J. Weber, 1981: UHF scintillation activity over polar latitudes. Geophys. Res. Lett., 8, 277-280, doi: 10.1029/GL 008i003p00277. [Link]

Born, M. and E. Wolf, 1980: Principles of Optics, $6^{\text {th }}$ edition, Pergamon, N. Y.

Fjeldbo, G., A. J. Kliore, and V. R. Eshelman, 1971: The neutral atmosphere of Venus as studied with the Mariner V radio 
occultation experiment. Astron. J., 76, 123-140, doi: 10.1086/111096. [Link]

Hajj, G. A. and L. J. Romans, 1998: Ionospheric electron density profiles obtained with the global positioning system: Results from the GPS/MET experiment. Radio Sci., 33, 175-190, doi: 10.1029/97RS03183. [Link]

Hajj, G. A., L. C. Lee, X. Pi, L. J. Romans, W. S. Schreiner, P. R. Straus, and C. Wang, 2000: COSMIC GPS ionospheric sensing and space weather. Terr. Atmos. Ocean. Sci., 11, 235-272.

Hocke, K. and K. Igarashi, 2002a: Structure of the Earth's lower ionosphere observed by GPS/MET radio occultation. J. Geophys. Res., 107, 1057, doi: 10.1029/2001JA900158. [Link]

Hocke, K. and K. Igarashi, 2002b: Electron density in the F region derived from GPS/MET radio occultation data and comparison with IRI. Earth Planets Space, 54, 947-954.

Immel, T. J., E. Sagawa, S. L. England, S. B. Henderson, M. E. Hagan, S. B. Mende, H. U. Frey, C. M. Swenson, and L. J. Paxton, 2006: Control of equatorial ionospheric morphology by atmospheric tides. Geophys. Res. Lett., 33, L15108, doi: 10.1029/2006GL026161. [Link]

Jakowski, N., A. Wehrenpfennig, S. Heise, Ch. Reigber, H. Lühr, L. Grunwaldt, and T. K. Meehan, 2002: GPS radio occultation measurements of the ionosphere from CHAMP: Early results. Geophys. Res. Lett., 29, 1457, doi: 10.1029/ 2001GL014364. [Link]

Kursinski, E. R., G. A. Hajj, S. S. Leroy, and B. Herman, 2000: The GPS radio occultation technique. Terr. Atmos. Ocean. Sci., 11, 53-114.

Lei, J., S. Syndergaard, A. G. Burns, S. C. Solomon, W. Wang, Z. Zeng, R. G. Roble, Q. Wu, Y. H. Kuo, J. M. Holt, S. R. Zhang, D. L. Hysell, F. S. Rodrigues, and C. H. Lin, 2007: Comparison of COSMIC ionospheric measurements with ground-based observations and model predictions: Preliminary results. J. Geophys. Res., 112, A07308, doi: 10.1029/ 2006JA012240. [Link]

Liu, L., H. Le, W. Wan, M. P. Sulzer, J. Lei, and M. L. Zhang, 2007: An analysis of the scale heights in the lower topside ionosphere based on the Arecibo incoherent scatter radar measurements. J. Geophys. Res., 112, doi: 10.1029/2007 JA012250. [Link]

Rishbeth, H. and O. K. Garriott, 1969: Introduction to ionospheric physics, 331 pp, Academic, San Diego, California, doi: 10.1126/science.168.3937.1336-a. [Link]

Rius, A., G. Ruffini, and A. Romeo, 1998: Analysis of Ionospheric Electron Density distribution from GPS/MET occultations. IEEE Trans. Geosci. Remote Sensing, 36, $383-$ 394, doi: 10.1109/36.662724. [Link]

Rocken, C., R. Anthes, M. Exner, D. Hunt, S. Sokolovskiy, R. Ware, M. Gorbunov, W. Schreiner, D. Feng, B. Herman, Y. H. Kuo, and X. Zou, 1997: Analysis and validation of GPS/MET data in the neutral atmosphere. J. Geophys. Res., 102, 29849-29866, doi: 10.1029/97JD02400. [Link]

Rocken, C., Y. H. Kuo, W. Schreiner, D. Hunt, S. Sokolovskiy, and C. McCormick, 2000: COSMIC system description. Terr. Atmos. Ocean Sci., 11, 21-52.

Schreiner, W. S., S. V. Sokolovskiy, C. Rocken, and D. C. Hunt, 1999: Analysis and validation of GPS/MET radio occultation data in the ionosphere. Radio Sci., 34, 949-966, doi: 10.1029/1999RS900034. [Link]

Stankov, S. M. and N. Jakowski, 2006: Topside ionospheric scale height analysis and modelling based on radio occultation measurements. J. Atmos. Sol.-Terr. Phys., 68, 134162, doi: 10.1016/j.jastp.2005.10.003. [Link]

Stankov, S. M., N. Jakowski, S. Heise, P. Muhtarov, I. Kutiev, and R. Warnant, 2003: A new method for reconstruction of the vertical electron density distribution in the upper ionosphere and plasmasphere. J. Geophys. Res., 108, 1164, doi: 10.1029/2002JA009570. [Link]

Stolle, C., N. Jakowski, K. Schlegel, and M. Rietveld, 2004: Comparison of high latitude electron density profiles obtained with the GPS radio occultation technique and EISCAT measurements. Ann. Geophys., 22, 2015-2022.

Straus, P. R., 2007: Ionospheric climatology derived from GPS occultation observations made by the ionospheric occultation experiment. Adv. Space Res., 39, 793-802. doi: 10.1016/j.asr.2006.08.009. [Link]

Tsai, L. C., W. H. Tsai, W. S. Schreiner, F. T. Berkey, and J. Y. Liu, 2001: Comparisons of GPS/MET retrieved ionospheric electron density andground based ionosonde data. Earth Planets Space, 53, 193-205.

Vladimer, J. A., P. Jastrezebski, M. C. Lee, P. H. Doherty, D. T. Decker, and D. N. Anderson, 1999: Longitude structure of ionospheric total electron content at low latitudes measured by the TOPEX/Poseidon satellite. Radio Sci., 34, 1239-1260, doi: 10.1029/1999RS900060. [Link] 